Conclusions There are occasions when hospital admission is appropriate. However, there was clear evidence showing support from the night team could prevent or postpone hospital admissions.

\section{THE VALUE OF A NIGHT SERVICE FOR HOSPICE AT HOME; REASONS FOR MAKING CONTACT AT NIGHT}

SG Spiro, A Ward, J Sixsmith, A Graham. Rennie Grove Hospice Care, The University of Northampton, The University of Dundee

\subsection{6/bmjspcare-2018-ASPabstracts.69}

Background People approaching the end of their lives have physical and psychological needs that should be met in a timely way, at any point during the night or day, and met in a way which addresses their needs and preferences. Crises at any time requires prompt, safe and effective urgent care (NICE 2011).

Methods Rennie Grove (RG) provides 24/7 care, with a night service available from $9.15 \mathrm{pm}$ to $7.15 \mathrm{am}$. RG conducted an independent study to identify the value of the service, including reasons people make contact at night and how many calls could be settled without a visit. Over the study period (145 nights) each call was charted with demographic details, reason for call and outcome.

Results 550 calls were received, resulting in 335 visits. On 7 nights there were no visits, the highest number in a night was 9, with an average 3.79 visits per night. Only those consenting to participate were analysed, reducing eligibility to 351 calls and 208 visits. Of these, reasons for a night visit included symptom control (64\%), carer support (19\%), death (13\%), death verified by night team (9\%), message (7.5\%), planned visit (7\%) and 'other' (4\%). Major symptoms included pain (42\%), anxiety (32\%), nausea (17\%) and a small number with chest problems, bowel issues, pyrexia, syringe pumps. In 143 cases a visit was not necessary due to telephone reassurance (55\%), another service being more suitable $(12 \%)$, the team were too busy $(8 \%)$, and $12 \%$ were handed to the next shift.

Conclusions The night team plays a vital role in $24 / 7$ care, with $40 \%$ of calls being managed without a visit. Visits were, in the main, for symptoms or problems concerning support. Death, for a Hospice at Home service also becomes a significant issue requiring a prompt and caring response.

\section{PERSONALISED CARE PLANNING TOOL FOR DYING PATIENTS; PEOPLES PERCEPTIONS AND IMPACT ON CARE}

Kate Tredgett, Karen Brown. Great Western Hospitals NHS Foundation Trust Swindon

\subsection{6/bmjspcare-2018-ASPabstracts.70}

Introduction In response to 'One chance to get it right' we developed a local tool to support the care of dying people in an acute hospital. It comprised a document with guidance and tear out elements for clinical staff and a patient/family information book. We report on the data acquired on the tool's impact on care and the perceptions of relatives and clinicians. Methods and results -Impact on care; This was evaluated through feedback from bereaved families using a validated questionnaire. Questionnaire responses were compared for the deaths supported and not supported by the tool. Overall score for quality of care from 123 completed questionnaires was $16 \%$ higher when care was supported by the tool $(72 \% \mathrm{v}$ $88 \%)$.

-Clinician Feedback; More than $75 \%$ of Trust staff have completed mandatory video based e.learning training in care of the dying and in using the tool. Feedback was collected from 60 medical and nursing staff of mixed grades following a learning event. $63 \%$ rated the tool 'very helpful' with the remainder rating it 'quite helpful'. Comments included that the tool provides 'written evidence of discussions' and 'staff are aware of the patient's wishes,' and highlighted practical issues including that it is necessarily extensive and takes a long time to complete'.

-Family Feedback; In addition to the completing the validated questionnaire, families were invited provide feedback on the tool itself. $96 \%$ of respondents rated it very or quite helpful with comments including 'discussed in detail' and 'I'm glad it was there'.

Discussion The findings suggest that the tool is helpful. Because deaths not supported by the tool may differ systematically from those supported by the tool, there may be bias which could dilute the tool's impact as evaluated by the questionnaire. Clinician and family feedback support its ongoing use and continued development.

\section{PRELIMINARY OUTCOME ANALYSIS OF INTEGRATED CARE FOR ADVANCED RESPIRATORY DISORDER (ICARE) - A MULTIDISCIPLINARY PALLIATIVE REHABILITATION PROGRAM FOR ADVANCED LUNG DISEASE IN A COMMUNITY HOSPITAL}

Nicholas Lee, Neo Han Yee. Lee Kong Chian School of Medicine Nanyang Technological University Singapore, Tan Tock Seng Hospital Singapore, Ren Ci Hospital Singapore

\subsection{6/bmispcare-2018-ASPabstracts.71}

Background Dyspnea is a prevalent and debilitating symptom, especially in patients with advanced pulmonary diseases. Dyspnea support services have been shown to improve functional outcomes and quality of life. This report presents a retrospective audit of a novel inpatient dyspnea support service for advanced respiratory diseases in Singapore.

Methods ICARE employs multidisciplinary dyspnea management, pulmonary rehabilitation, structured comorbidity screening using CO-morbidity Assessment Template (COAT), and dyspnea self-management interventions for patients discharged from Respiratory Medicine. The primary outcomes assessed were 6 min Walk Distance (6MWD) and Modified Barthel Index (MBI). Secondary outcomes include clinical issues identified by COAT that were treated, as well as 30 day readmissions.

Results ICARE had 39 unique admissions with 4 recurrent patients. The patients were mostly elderly Chinese males who were exceptionally frail with considerable co-morbidity. Their mean age was $73.2( \pm 9.5)$ years, $87.1 \%$ had COPD of which $81.8 \%$ was GOLD stage 3/4. Admission 6MWD was $130.4 \mathrm{~m}$ ( \pm 90.6$) .3 .3( \pm 1.3)$ of 5 basic ADLs were affected by dyspnea. They had mean 5.1 respiratory hospitalisations 1 year prior to ICARE admission with median hospital LOS 30.0 (IQR 20.0-60.0) days, 12.8\% had 10 hospitalisations.

The median program duration was 24.0 (IQR 12-35) days. 6MWD improved by median $30 \mathrm{~m}$ (IQR 20-60) $(\mathrm{p}=0.05)$. 